\title{
Right insular damage decreases heartbeat awareness and alters cardio- visual effects on bodily self-consciousness
}

\author{
Roberta Ronchi ${ }^{\mathrm{a}, \mathrm{b}, *}$, Javier Bello-Ruiz ${ }^{\mathrm{a}, \mathrm{b}}$, Marta Lukowska ${ }^{\mathrm{a}, \mathrm{c}}$, Bruno Herbelin ${ }^{\mathrm{a}, \mathrm{b}}$, \\ Ivan Cabrilo ${ }^{\mathrm{d}}$, Karl Schaller ${ }^{\mathrm{d}}$, Olaf Blanke ${ }^{\mathrm{a}, \mathrm{b}, \mathrm{e}}$ \\ a Laboratory of Cognitive Neuroscience, Brain Mind Institute, School of Life Sciences, Ecole Polytechnique Fédérale de Lausanne, Lausanne, Switzerland \\ ${ }^{\mathrm{b}}$ Center for Neuroprosthetics, Ecole Polytechnique Fédérale de Lausanne, Lausanne, Switzerland

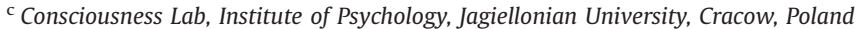 \\ ${ }^{\mathrm{d}}$ Neurosurgery Division, Department of Clinical Neurosciences, Geneva University Hospitals, Geneva, Switzerland \\ e Neurology Division, Department of Clinical Neurosciences, Geneva University Hospitals, Geneva, Switzerland
}

\section{A R T I C L E I N F O}

\section{Article history:}

Received 23 July 2014

Received in revised form

3 February 2015

Accepted 9 February 2015

Available online 10 February 2015

Keywords:

Bodily self-consciousness

Interoception

Multisensory

Insula

Brain damage

\begin{abstract}
A B S T R A C T
Recent evidence suggests that multisensory integration of bodily signals involving exteroceptive and interoceptive information modulates bodily aspects of self-consciousness such as self-identification and self-location. In the so-called Full Body Illusion subjects watch a virtual body being stroked while they perceive tactile stimulation on their own body inducing illusory self-identification with the virtual body and a change in self-location towards the virtual body. In a related illusion, it has recently been shown that similar changes in self-identification and self-location can be observed when an interoceptive signal is used in association with visual stimulation of the virtual body (i.e., participants observe a virtual body illuminated in synchrony with their heartbeat). Although brain imaging and neuropsychological evidence suggest that the insular cortex is a core region for interoceptive processing (such as cardiac perception and awareness) as well as for self-consciousness, it is currently not known whether the insula mediates cardio-visual modulation of self-consciousness. Here we tested the involvement of insular cortex in heartbeat awareness and cardio-visual manipulation of bodily self-consciousness in a patient before and after resection of a selective right neoplastic insular lesion. Cardio-visual stimulation induced an abnormally enhanced state of bodily self-consciousness; in addition, cardio-visual manipulation was associated with an experienced loss of the spatial unity of the self (illusory bi-location and duplication of his body), not observed in healthy subjects. Heartbeat awareness was found to decrease after insular resection. Based on these data we propose that the insula mediates interoceptive awareness as well as cardio-visual effects on bodily self-consciousness and that insular processing of interoceptive signals is an important mechanism for the experienced unity of the self.
\end{abstract}

(c) 2015 Elsevier Ltd. All rights reserved.

\section{Introduction}

The study of the bodily features of self-consciousness is an intriguing topic in recent neuroscience. Normally, people experience a unitary sense of self that is felt to be localized within the boundaries of the physical body (bodily self-consciousness, BSC; Blanke, 2012; de Vignemont, 2011). Multiple sources of exteroceptive information (tactile, vestibular, visual signals) contribute to BSC. Indeed, if one or more of these signals are manipulated different components of BSC can be altered experimentally, such as the feeling that this body belongs to me (self-identification or body

\footnotetext{
* Corresponding author at: Laboratory of Cognitive Neuroscience, Brain Mind Institute, School of Life Sciences, Ecole Polytechnique Fédérale de Lausanne, Lausanne 1015, Switzerland.

E-mail address: roberta.ronchi@epfl.ch (R. Ronchi).
}

ownership) and the feeling of where my body is located in space (self-location) (Blanke and Metzinger, 2009). One example of such an experimental paradigm is the so-called Full Body Illusion (FBI). During this illusion people view a virtual body being stroked while they simultaneously feel touch on their own body: after several minutes of stimulation this procedure induces the feeling that the virtual body belongs to them (self-identification) and is associated with a recalibration of where subjects perceive to be localized in space, characterized by a drift in self-location towards the virtual body (Aspell et al., 2009; Ehrsson, 2007; Lenggenhager et al., 2007; Palluel et al., 2011; Petkova and Ehrsson, 2008). If the visual and tactile stimuli are administered out of synchrony, no changes in BSC occur. Thus, during the FBI two temporally synchronous exteroceptive (vision and touch) stimuli are in spatial conflict: each stimulation refers to a body, and the bodies (the real and the virtual bodies) occupy two different spatial locations. 
Exteroceptive information is not the only source contributing to BSC. It has been theorized that internal states and visceral signals are at least of equal importance for the bodily self (Craig, 2002; Damasio, 2000). Moreover, recent behavioural experiments (Aspell et al., 2013; Suzuki et al., 2013) demonstrated that exteroceptive and interoceptive signals jointly affect BSC. Thus, Aspell et al. (2013) showed that the FBI can also be induced by using a cardio-visual (CV) conflict instead of the visuo-tactile (VT) conflict as described above. Using a novel FBI-CV paradigm, during which participants view a virtual body that is surrounded by a glow flashing in synchrony with the subjects' heartbeat, it was reported that both self-identification for and drifts in self-location towards the virtual body are induced similarly to changes as observed by VT stimulation (Aspell et al., 2013). A similar procedure was shown to induce self-attribution of a virtual hand after CV synchronous stimulation in a CV version of the rubber hand illusion (Suzuki et al., 2013). The effects induced by this novel CV manipulation suggest that interoceptive (cardiac) and exteroceptive (visual) signals are integrated and that both signals and their integration are relevant for BSC.

However, the brain mechanisms of CV integration in general and during the FBI-CV in particular are currently unknown. One key candidate region that has been repeatedly associated with the processing of interoceptive cardiac signals using functional magnetic resonance imaging (fMRI) is the insular cortex (Critchley et al., 2004; Wiebking et al., 2014). Moreover, awareness of interoceptive signals, as measured by a heartbeat awareness task, has been related to activity of the right insula (Critchley et al., 2004; Zaki et al., 2012). Other evidence has linked the insula to bodily self-processing by associating insular activity with illusory self-attribution of a fake hand (Tsakiris et al., 2007). Neuropsychological studies also support the link between insula and BSC. Patients with right brain damage may show impaired selfattribution of their own body parts (somatoparaphrenia: see Vallar and Ronchi, 2009 for a review). The neural correlates of somatoparaphrenia include an extensive network of fronto-temporoparietal areas, with the involvement of subcortical structures; some cases have been reported with lesion affecting deep cortical regions such as the insula (Cereda et al., 2002; Karnath and Baier, 2010; Levine et al., 1991). Moreover, patients with heautoscopy, illusory own body perceptions characterized by the feeling of seeing one's own body in extrapersonal space and strong selfidentification with this illusory body, suffer from lesions centred in the insular region (Heydrich and Blanke, 2013).

In the present study we investigated the involvement of insular cortex in heartbeat awareness and the effects of cardio-visual stimulation on BSC in a patient before and after resection of a selective right neoplastic insular lesion.

\section{Materials and methods}

\subsection{Participants}

The patient was a 43-year-old male, working as pastry chef. He was admitted to University Geneva Hospital for an epileptic generalized seizure. The computerized tomography (CT) and the magnetic resonance imaging (MRI) scans showed the presence of a right neoplastic lesion, affecting the entire right insula. The lesion invaded both anterior and posterior lobules of the right insula with the exception of the caudal portion of the anterior long insular gyrus. It was in general circumscribed by the anterior, superior and inferior peri-insular sulci. However, antero-inferiorly it extended beyond the insula's transverse gyrus to the posteromedial orbital lobule of the frontal lobe; inferiorly, it spread into the planum polare of the temporal lobe; medially, it involved the capsula extrema, the claustrum, the capsula externa and abutted the putamen along its whole lateral length, as far cranially as the superior-lateral aspect of the head of the caudate nucleus. Interviews with the patient revealed that the first symptoms consisted of short euphoric states that started several months before the current hospitalization. During these states he smiled and laughed without any apparent reason or associated mirth. No other abnormal sensations or behaviours were noted. At the time of hospitalization in the Department of Neurosurgery at Geneva Hospital the patient was alert and cooperative. The neurological exam showed preserved motor, somatosensory and visual functions, which remained unimpaired also after the tumour removal. No visual or tactile extinction to double stimulation was detected. A short neuropsychological evaluation was administered to the patient. He was perfectly oriented in time (day, month, year, season, date) and in space (country, canton, city, place, floor), and completely aware about his medical/neurosurgical condition (as assessed during a clinical interview). With respect to the spatial functions, we administered to the patient two cancellation tasks and one bisection task, as classically done in standard clinical evaluation of unilateral left spatial neglect. Both before and after the surgery the patient demonstrated a good performance and within the cut off scores of the control sample: no target omission in the star cancellation test, only one omission on the left side in the letter cancellation test after surgery, and a deviation score of $-1.67 \mathrm{~mm}$ before and $+0.5 \mathrm{~mm}$ after the surgery. No left personal neglect was detected, asking the patient to reach a body part on the left side using the unimpaired right hand (score: 0/3). Finally, during a semi-structured interview the patient demonstrated a perfect and immediate attribution of the left and right body-parts both before and after the operation, therefore no signs of somatoparaphrenia (Vallar and Ronchi, 2009). Table 1 shows the results of the neuropsychological assessment of left spatial and personal neglect. Fig. 1 shows the magnetic resonance images of the patient's brain damage before (Fig. 1A) and after (Fig. 1B) the

Table 1

Neuropsychological evaluation of the patient to assess the presence of left spatial and personal neglect.

\begin{tabular}{llll}
\hline Test & Cut off (range) & $\begin{array}{l}\text { Patient's score } \\
\text { Before surgery }\end{array}$ & After surgery \\
\hline $\begin{array}{l}\text { Unilateral spatial neglect } \\
\text { Target cancellation }\end{array}$ & & & \\
Star $^{\mathrm{a}, \mathrm{b}}$ & & 0 & 0 \\
Letter $^{\mathrm{c}, \mathrm{d}}$ & $2(0 / 56)$ & 0 & 1 \\
Line bisection $^{\mathrm{b}}$ & $+0.74^{\#}(-2.3 /$ & -1.67 & +0.5 \\
& $+5.67)$ & & \\
& & & 0 \\
$\begin{array}{l}\text { Personal neglect } \\
\text { Reaching of body parts }\end{array}$ & $0(0 / 3)$ & 0 & \\
Hand $^{\mathrm{e}}$ & & & \\
\end{tabular}

Scores: cancellation tasks (differences of omissions between the left and the right side of the paper); line bisection (deviation in millimetres: positive score=rightward deviation; negative score=leftward deviation); hand reaching (errors).

\# Mean bisection deviation of controls.

a Wilson, B., Cockburn, J., Halligan, P.W., 1987. Behavioural Inattention Test Thames Valley Test Company, London.

${ }^{b}$ Ronchi, R., Posteraro, L., Fortis, F., Bricolo, E., Vallar, G., 2009. Perseveration in left spatial neglect: drawing and cancellation tasks. Cortex 45(3), 300-331

c Diller, L., \& Weinberg, J., 1977. Hemi-Inattention in rehabilitation. The evolution of a rational remediation program. In: Weinstein, E. A., Friedland, R.P. (Eds.), Hemi-inattention and Hemisphere Specialization. Raven Press, New York, pp. 6282.

d Vallar, G., Rusconi, M.L., Fontana, S., Musicco, M., 1994. Tre test di esplorazione visuo-spaziale: Taratura su 212 soggetti normali. Arch. Psicol. Neurol. Psichiatr. 55 (4), 827- 841

e Bisiach, E., Perani, D., Vallar, G., Berti, A., 1986. Unilateral neglect: personal and extrapersonal. Neuropsychologia 24(6), 759-767. 

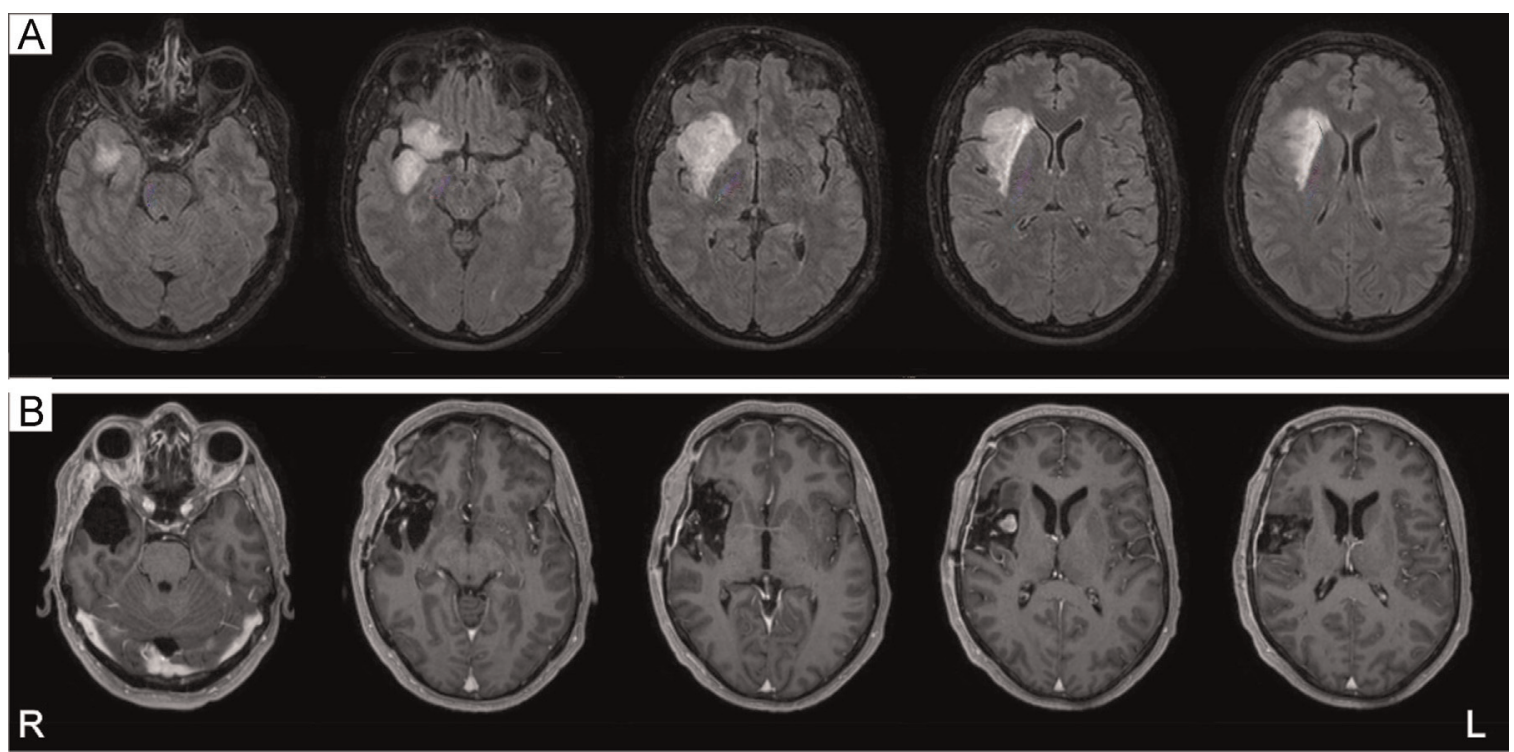

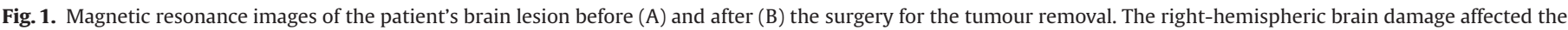
entire insula (anterior-middle and posterior areas). R: right; L: left.

operation for an oligoastrocytoma (stage II) as confirmed by pathology: the post-operative control MRI revealed a near-to-complete resection with a small tumour residuum lateral to a foremost tip of the frontal horn of the right lateral ventricle.

A group of 16 age-matched neurologically unimpaired participants (6 males; mean age: 42.4, SD: \pm 3.9 , range: $35-50$ ) were also tested as control (C) group. All participants had normal or corrected to normal vision and were naïve to the purpose of the study and the experimental manipulation induced. All participants gave their written informed consent for the study's participation.

\subsection{Experimental setup}

Patient and controls underwent the FBI paradigm under CV stimulation and consisted of the virtual reality setup that has been described previously (Aspell et al., 2013). The virtual reality setup was composed of two cameras placed on a tripod that were positioned two metres behind the participant. The cameras filmed the back of the participant and the recorded video of his body was projected in real time onto the head mounted display (HMD VR1280 Virtual Research Systems, Inc.; Dual SXGA $1280 \times 1024$ resolution, $60^{\circ}$ diagonal field of view). The HMD was covered by black tissue occluding surrounding visual input. Moreover, during the whole experimental session the participant listened to white noise presented through headphones, to exclude surrounding auditory input. An in-house software (ExpyVR, http://lnco.epfl.ch/ expyvr) was used for visual stimulus presentation and real-time synchronization.

During the FBI, the participant saw his body on the HMD and the body was illuminated periodically by a glow surrounding the body and flashing on and off with the heartbeat. The subject's electrocardiogram (ECG) was recorded for the entire session. Raw data were acquired by an Arduino ${ }^{\mathrm{TM}}$ microcontroller with a mounted e-Health Sensor Shield V2.0 from Libelium ${ }^{\mathrm{TM}}$ through three electrodes placed on the chest. In-house software detected, in real time, the $\mathrm{R}$ peak of the QRS complex (amplitude change over $80 \%$ of the difference between the exponential moving averages of the maxima and minima of the raw signal). The flashing glow lasted for $500 \mathrm{~ms}$ (sinusoidal transparency from $0 \%$ to $100 \%$ visible).

In the synchronous condition, the visual glow flashed synchronously with the QRS complex (with a maximum delay of
16 ms after detection of the R peak). During asynchronous cardiovisual stimulation, the virtual body was illuminated at a frequency equal to $80 \%$ or $120 \%$ of the actual heart rate (as in Aspell et al., 2013). This was obtained by replaying at controlled speed a prerecorded ECG (30 s template from a healthy subject): an artificial heartbeat flashing was therefore simulated in real-time to emulate a heartbeat at either $80 \%$ (slower) or $120 \%$ (faster) of the continuously measured heart rate of the participant. For the patient, the $80 \%$ asynchronous condition was used.

An heartbeat awareness task (Schandry, 1981) was administered to check for the level of interoceptive awareness of participants (see below for more details).

\subsection{Procedure}

The FBI-CV included two conditions: CV synchronous (S) and $\mathrm{CV}$ asynchronous (AS) stimulation. The sequence of tests in each condition was organized as follows: first we tested a baseline selflocation (see below), followed by the cardio-visual FBI exposure ( $2 \mathrm{~min}$ ), then the experimental self-location followed by the administration of the questionnaire. Patient and control subjects repeated the whole procedure twice. After synchronous and asynchronous cardio-visual stimulation, we administered a heartbeat awareness task. The patient was tested three weeks before and three months after the resective surgery (A-B experimental assessments); control participants also performed the procedure twice (Day1-Day2), with about two weeks of timeinterval.

\subsection{Behavioural measures}

Before and after each FBI-CV session, a task traditionally used to assess self-location was administered: participants were moved backwards for $1.5 \mathrm{~m}$ and then they have to walk back to the initial position (see details in Aspell et al., 2013; Lenggenhager et al., 2007). This position (i.e., the position from where they started walking backwards) was the same position in which they were placed during the induction of the FBI. The distance between the position estimated by the patient and his real original position was measured in centimetres. Three trials were administered for each repetition of the task (pre- and post-FBI). The accuracy in the selflocation task was computed by the average of the three trials of 
Table 2

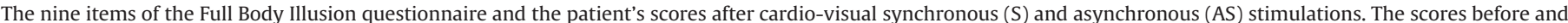
after the surgery are shown.

\begin{tabular}{|c|c|c|c|c|}
\hline \multirow[t]{3}{*}{ Item } & \multirow{2}{*}{\multicolumn{2}{|c|}{$\begin{array}{l}\text { Patient's score } \\
\text { Before surgery }\end{array}$}} & \multirow{2}{*}{\multicolumn{2}{|c|}{$\begin{array}{l}\text { Patient's score } \\
\text { After surgery }\end{array}$}} \\
\hline & & & & \\
\hline & $\mathbf{S}$ & AS & $\mathbf{S}$ & AS \\
\hline \multicolumn{5}{|l|}{ During the experiment there were times when: } \\
\hline Q1: It seemed as if I was feeling my heartbeat where I was seeing the semi-transparent template flashing & -3 & -3 & -2 & -3 \\
\hline Q2: I felt as if my heart was in the virtual body & -3 & -3 & -3 & -3 \\
\hline Q3: I felt as if the virtual body was my body & +3 & +3 & +3 & -3 \\
\hline Q4: It felt as if my body was drifting towards the virtual body & -3 & -3 & -3 & -3 \\
\hline Q5: It seemed as if I might have more than one body & +3 & -3 & -3 & -3 \\
\hline Q6: It seemed as if I was having two hearts & -3 & -3 & 0 & -3 \\
\hline Q7: It appeared (visually) as if the virtual body was drifting backwards (towards my body) & -3 & -3 & -3 & -3 \\
\hline Q8: It seemed as though I was in two places at the same time & +3 & -3 & +3 & -3 \\
\hline Q9: It seemed as if the flashing semi-transparent template was my heartbeat & -3 & -3 & -3 & -3 \\
\hline
\end{tabular}

each task repetition, measured in centimetres with respect to the objective starting position (corresponding to 0 ): negative numbers indicated underestimated positions (i.e., the real body was located more distant from the virtual body) while positive numbers indicated overestimated positions (i.e., the real body was located closer to the virtual body). The difference between the mean postand pre-measures was computed to obtain a measure of self-location drift for each condition.

After the self-location task, the questionnaire as used by Aspell et al. (2013) was administered to investigate self-identification with the virtual body and other subjective sensations (see Table 2). The patient and control subjects were asked to indicate agreement or disagreement for nine questions $(\mathrm{Q})$ using a 7-point coloured vertical Likert scale, ranging from a complete disagreement (the bottom extreme, red point, scored by the examiner as -3 ) to a complete agreement (the top extreme, green point, scored by the examiner as +3 ).

After the questionnaire, the heartbeat detection task as originally proposed by Schandry (1981) was administered as a measure of interoceptive heartbeat awareness. Patient and control participants were asked to report the number of heartbeats they detected during four fixed time intervals $(25 \mathrm{~s}, 35 \mathrm{~s}, 45 \mathrm{~s}, 100 \mathrm{~s}$, given in randomized order), and we compared their score with the real number of heartbeats (recorded by the ECG) using the following formula: $1 / 4 \sum$ [1-(|recorded heartbeats-counted heartbeats|)/recorded heartbeats]. Based on this, the interoceptive sensitivity score ranged from 0 to 1 , with higher scores indicating small differences between recorded and counted heartbeats (i.e. high interoceptive sensitivity) and lower scores indicating big differences between recorded and counted heartbeats (i.e. low interoceptive sensitivity).

\subsection{Statistical analyses}

With respect to the performance of the healthy age-matched participants, parametric analyses ( $t$-test) for scores in the self-location task and the heartbeat awareness task were used; nonparametric analyses (Wilcoxon signed ranks test; Siegel and Castellan, 1988) were applied to the questionnaire ratings (which were not normally distributed). Spearman non-parametric correlation coefficients were computed between variables of interest. To compare the scores of the insular patient with the corresponding mean data from the control group, we have used the single case Crawford tests, implemented in the software SINGSLIMS.exe (see Crawford and Garthwaite, 2002: http://homepages.abdn.ac.uk/j. crawford/pages/dept/psychom.htm\#conflims. See a similar use of the Crawford test in Bolognini et al., 2014). The software tests whether an individual's score is significantly different from a control/normative sample and provides a point estimate of the rarity/abnormality of the score; namely, it estimates the percentage of the population that would obtain a lower score. Moreover, the method provides $95 \%$ confidence limits on this percentage. This approach is commonly used in neuropsychological single case studies, as it allows comparing a given individual's score with a control sample with modest size. This method applies a modified $t$-test in which the individual (i.e., the patient in our case) is treated as a sample of mean $=1$ and, therefore, does not contribute to the estimate of the within group variance. Additionally, with respect to the results of the questionnaire, we have performed the single case Crawford tests using ipsatized scores, which are standard scores calculated with individual subject's mean and standard deviation. Statistical analyses using ipsatized scores allow both a comparison free of response biases and a proper use of parametric tests as the data are normalized (Broughton and Wasel, 1990; Cattell, 1944; see a previous use of ipsatized scores on questionnaire ratings in Romano et al., 2014). The significance (alpha) level used was 0.05 .

\section{Results}

\subsection{Questionnaire}

Table 2 reports the list of questions and the patient's scores of the questionnaire items before and after the surgery. Before the operation, the FBI-CV induced maximal self-identification with the virtual body in the $\mathrm{S}$ and in the AS conditions (Q3-S and -AS: +3). Similar high ratings were given for the feeling of having more than one body (illusory reduplication; Q5-S: +3 ) and the sensation to be in two places at the same time (illusory bi-location; Q8-S: +3 ): however, these sensations of illusory reduplication and illusory bilocation were induced only in the CV-S condition, not in the CV-AS condition. All other questions in S (Q1, Q2, Q4, Q6, Q7, Q9) and AS (all questions except for Q3) stimulations were rated in total disagreement (all scores: -3 ). We note that after the S stimulation condition, the patient spontaneously reported a strange sensation and expressed to have felt as if he "had a twin" and as if the virtual body had been his "twin".

In the control group, no questionnaire item showed a significant difference between $\mathrm{S}$ and AS conditions on the first (Wilcoxon signed ranks test; all ps $>0.14$ ) or the second (all $p s>0.34$, only for Q5 $p>0.07$, with the mean score in AS slightly higher than $\mathrm{S}$, but both strongly negative) day. Moreover, no difference between the scores of day1 and day2 was found 
(all $p s>0.11$ ): therefore, we averaged the behavioural scores of the two experimental sessions in controls, and we compared the mean values with the patient's questionnaire scores (difference S-AS) pre-surgery (Crawford and Garthwaite, 2002). We found a significant difference for illusory reduplication (Q5; $t=6.093$, onetailed $p<0.001)$ and illusory bi-location (Q8; $t=4.447$, one-tailed $p<0.001$ ), but not for self-identification (Q3, $t=0.274$, one-tailed $p=0.394)$. However, for Q3 there was a tendency towards significance (patient vs. controls) in the $S(t=1.549$, one-tailed $p=0.071)$ but not in AS $(t=1.305$; one-tailed $p=0.106)$ condition, and the self-identification ratings (average for S and AS of Q3) tended to be greater in the patient than in controls $(t=1.511$, onetailed $p=0.076)$. No significant difference between patient and controls was found for any of the other questions (all $p s>0.38$ ). The same statistical significant results comparing patient's and controls data were also found using ipsatized scores (Q5; $t=5.555$, one-tailed $p<0.001$. Q8; $t=2.631$, one-tailed $p=0.001$; all other ps $>0.13$ ).

After the surgery, self-identification with the virtual body was only found during the $S$ stimulation (Q3-S: + 3; Q3-AS: -3 ). In the $\mathrm{CV}-\mathrm{S}$ condition the patient still reported strong illusory bi-location (Q8: + 3; same effect evoked in the pre-surgery CV-S), but no illusory body reduplication (Q5-S and -AS: -3 ). He now reported an intermediate agreement with the sensation of having two hearts (Q6: 0). All other questions for CV-S and CV-AS obtained negative scores (Q1-S: -2 ; all other scores: -3 ). The comparison with respect to the control group (difference $S-A S$ ) revealed a significant difference for self-identification (Q3; $t=4.030$, one-tailed $p=0.001$ ), for bi-location (Q8; $t=4.447$, one-tailed $p<0.001$ ), and for duplication of the heart (Q6; $t=2.463$, one-tailed $p=0.013$ ). No significant difference for any of the other questions was found (all $p s>0.35$ ). The same statistical significant results comparing patient's and controls data were also found using ipsatized scores (Q3; $t=4.736$, one-tailed $p<0.001$. Q6; $t=2.707, p=0.008$. Q8; $t=3.411$, one-tailed $p=0.002$; all other $p s>0.34$ ).

Fig. 2 shows the patient's scores for the most relevant items of the questionnaire administered before (A) and after (B) the surgery, with the range (horizontal bars) corresponding to the confidence interval (95\%) of the control group.

\subsection{Self-location}

The patient showed a larger drift in self-location towards the virtual body in the $\mathrm{S}$ vs. AS condition both before and after the operation. Thus, before the surgery, the FBI-CV induced in the patient a mean positive drift (i.e., difference between post- and pre-FBI) in the $S$ condition $(+28.2 \mathrm{~cm})$ and a negative drift in the AS condition $(-13.8 \mathrm{~cm}$; difference $\mathrm{S}-\mathrm{AS}$ : $42.0 \mathrm{~cm})$. After the surgery, the same pattern was found (drift CV-S: $+12.3 \mathrm{~cm}$; drift CV-AS: $-22.2 \mathrm{~cm}$; difference S-AS: $34.5 \mathrm{~cm}$ ).

In the control group, the mean drift scores on day1 in the CV-S $(+2.25 \mathrm{~cm}, \mathrm{SD}: 16.1)$ and $\mathrm{CV}-\mathrm{AS}(+1.80 \mathrm{~cm}$; SD: 17 ; difference S-AS: $+0.45 \mathrm{~cm})$ conditions were both positive without a significant difference between $S$ and AS stimulations (paired $t$-test; $t=0.08, p=0.933$ ); a similar statistical result was found on day2 (CV-S: $-2.89 \mathrm{~cm}, \mathrm{SD}: 10.2$; CV-AS: $-1.31 \mathrm{~cm}, \mathrm{SD}$ : 9.7; difference S-AS: $-1.58 \mathrm{~cm})(t=-0.454, p=0.656)$. No difference between the scores of day 1 and day 2 was detected $(p=0.19)$ and we compared the mean values of day 1 and day 2 of the control group with the patient's self-location scores. This analysis revealed a significant difference between patient and controls in self-location (difference $S-A S)$ before $(t=2.884$, one-tailed $p=0.006)$ and after $(t=2.379$, one-tailed $p=0.016)$ the operation. Fig. 3 shows the patient's mean values of the drift in self-location before $(A)$ and after (B) surgery, with the range (horizontal bars) corresponding to the confidence interval (95\%) of the control group.

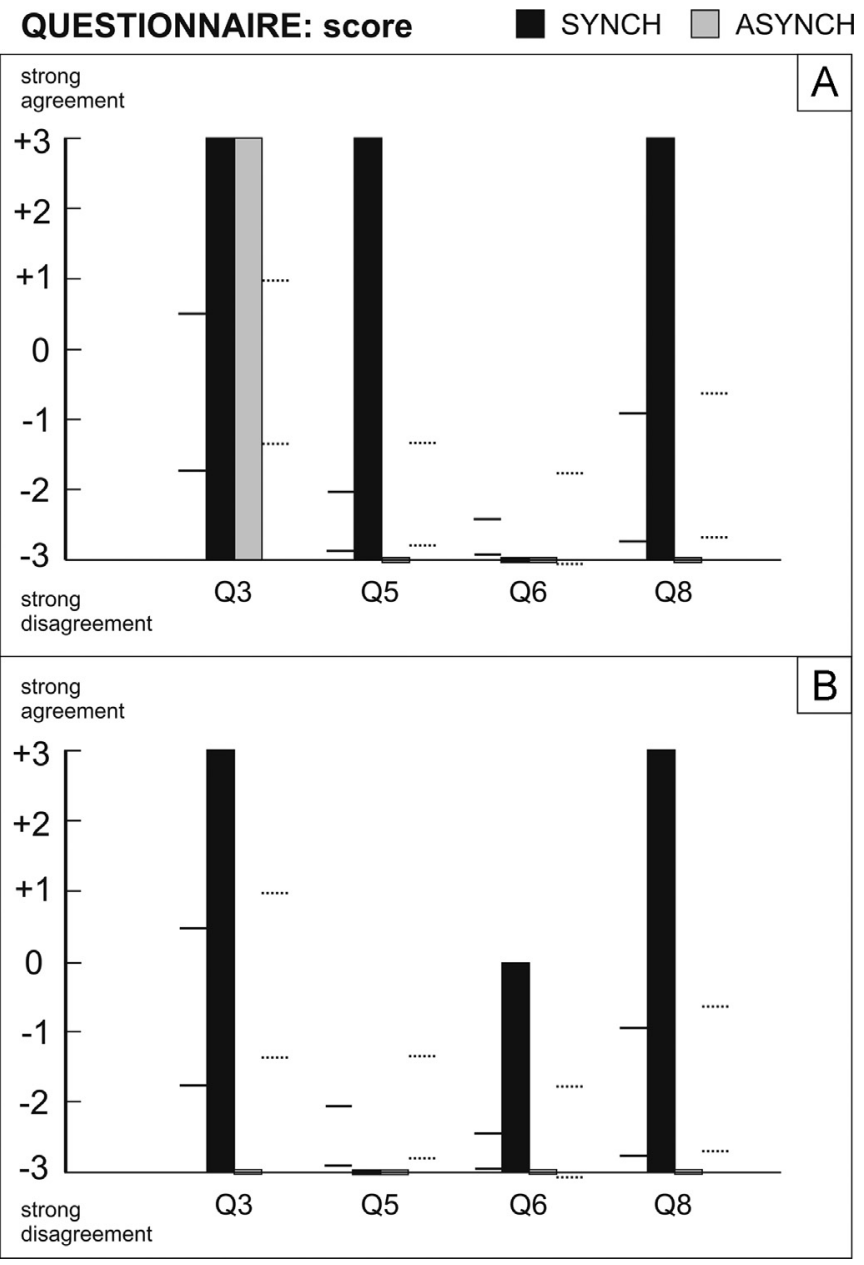

Fig. 2. Patient's agreement scores of some relevant questionnaire's items before (A) and after (B) the surgery. The scores ranged from the maximum disagreement $(-3)$ to the maximum agreement $(+3)$. The black continuous and dotted lines indicated the confidence interval of the control group at the $95 \%$ confidence level for synchronous and asynchronous conditions, respectively. The list of the questions and the values relative to each question's answer by the patient are reported in Table 2.

\section{SELF-LOCATION DRIFT (POST-PRE)}

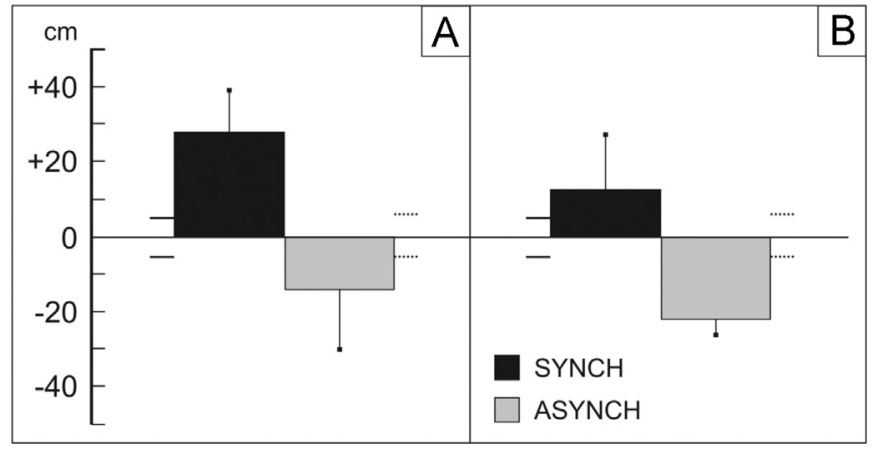

Fig. 3. Mean scores of the patient in the self-location task administered before (A) and after (B) the surgery. The drift indicates, in centimetres (SEM), the mean difference post-pre FBI. The black continuous and dotted lines indicated the confidence interval of the control group at the 95\% confidence level for synchronous and asynchronous conditions, respectively.

We note that with respect to previous research about alteration of BSC as induced by cardio-visual stimulation, [both when the body (Aspell et al., 2013) or only the hand (Suzuki et al., 2013) are involved], the present control group did not show the expected synchrony-dependent effect of self-identification with 
and self-location towards the virtual body. Several factors may account for this difference. First, our sample is age-matched with the patient, and thus much older than previously tested populations of healthy subjects. We note that increase in age has been shown to affect multisensory integration and peri- and extrapersonal space representation (Bloesch et al., 2013; Caçola et al., 2013; Ghafouri and Lestienne, 2000; Lepelley et al., 2010; Mahoney et al., 2011). Even if these cited studies included participants older than the present age-matched participants, the findings are in line with the idea that increased age affects the integration of multisensory information. Accordingly, the present control group could show reduced multisensory perception and thus reduced interoceptive-exteroceptive effects as tested in the present study. Moreover, the results of previous studies seem to suggest that the effect size of BSC alteration is smaller for cardio-visual than visuotactile stimulation (Aspell et al., 2013; see Suzuki et al., 2013 for a direct comparison of the two modalities). Finally, differences could also be related to the detection of the cardio-visual feedback during the FBI stimulation (see correlation analyses in Suzuki et al., 2013). The combination of these factors (demographic variable, cardio-visual effect size, heartbeat awareness) may have reduced the interoceptive-exteroceptive effects in our control group.

\subsection{Heartbeat detection}

The patient's heartbeat awareness score decreased from 0.82 (SD: 0.11) before the operation to 0.61 (SD: 0.16) after the operation. This change was absent in the control subjects and no difference was found between day1 $(M=0.69$; median $=0.63$; SD: $0.21)$ and day2 $(M=0.74 ;$ median $=0.77 ; \mathrm{SD}: 0.10)(t=-0.783$, $p=0.446$ ). For one control participant (C2) we failed to record the score on day1 due to a technical problem.

A first comparison between the patient's heartbeat awareness with the control group (average of day1 and day2) revealed no significant difference (pre-surgery: $t=0.586$, one-tailed $p=0.283$; post-surgery: $t=-0.657$, one-tailed $p=0.261)$. However, when we split the control participants in one subgroup with high $(N=8$, $M=0.85)$ and another with low $(N=8, M=0.59)$ heartbeat awareness (based on the median heartbeat awareness score of the control group; median $=0.72$ ) differences were found. Thus, we found that before the operation the patient's interoceptive awareness was comparable with the high-awareness control group ( $t=-0.377$, one-tailed $p=0.359$ ), but after the surgery significantly lower than this group $(t=-2.852$, one-tailed $p=0.012)$. The opposite trend was found when comparing the patient's heartbeat awareness with the low awareness group (pre-surgery: $t=1.963$, one-tailed $p=0.045$; post-surgery: $t=0.163$, one-tailed $p=0.438$ ). These findings are compatible with a decrease in heartbeat awareness due to the operation. The heart rate of the patient, recorded during the heartbeat awareness task, was $65.8 \mathrm{bpm}$ before and $64.8 \mathrm{bpm}$ after the surgery, respectively. The difference between the scores of heartbeat awareness cannot be explained by differences in heart rate between pre- and postsurgery, as they were almost identical (variance $=1.66$ across the four trials and two conditions).

Finally, in the control group we tested for correlation between the level of heartbeat awareness and both the measures of selflocation (drift) and self-identification (Q3: "I felt as if the virtual body was my body"). For heartbeat awareness we selected two different measures: (1) the score of the heartbeat detection task and (2) the rating of the questionnaire item about the recognition of the heartbeat in the flashing template during the FBI (Q9: "It seemed as if the flashing semi-transparent template was my heartbeat"). As there was no difference between day1 and day2, we used the average scores of both sessions and we performed a series of Spearman non-parametric correlation analyses on the mean scores. We found a trend towards a significant positive correlation between self-identification (Q3, difference S-AS) and the score on item Q9 (difference $\mathrm{S}-\mathrm{AS})(r=0.441, p=0.087)$. No correlation was found between self-location (difference $S-A S$ ) and Q9 (difference $S-A S)(r=0.001, p=0.996)$. Analyses between the measure of the heartbeat detection task with self-location (drift; $r=0.016, p=0.953$ ) or with self-identification (item Q3; $r=0.365, p=0.165$ ) showed no significant correlations.

\section{Discussion}

In the present study we used cardio-visual stimulation and describe the association of a decrease in heartbeat awareness with a pathologically enhanced modulation of BSC in a patient suffering from a lesion affecting the right insula. During the cardio-visual FBI paradigm the patient showed not only stronger self-identification with the virtual body, but also experienced an illusory duplication of his body and bi-location (he felt "as though I was in two places at the same time"). Both latter sensations were absent in the control subjects and are rare and highly unusual experiences that are normally not encountered in FBI paradigms. These findings were further corroborated by the patient's spontaneous report of having felt, during synchronous cardio-visual stimulation, as if he had a twin (i.e., the virtual body). Even if it has been demonstrated that it is possible to experimentally induce the feeling of having multiple bodies (Heydrich et al., 2013), this phenomenon has been recorded only when participants actually did see two bodies in front of them, that were visually "stroked", and only when visuo-tactile stimulation was applied synchronously. There were no differences for other sensations between the subjective questionnaire ratings of the patient vs. controls (i.e., items related to the recognition of the heartbeat in the flashing template/the feeling of heart displacement (Q1, Q2 and Q9) or the subjective alteration of self-location towards the virtual body (Q4 and Q7)). These items are considered control questions, at least in neurologically unimpaired subjects (Aspell et al., 2013), revealing the selective effect in the present insular patient. In addition to these subjective responses to the questionnaire's items, the patient also showed an objective synchrony-dependent drift in self-location task towards the location of the virtual body.

What brain mechanisms may mediate these cardio-visual effects on BSC? Most earlier studies investigated the brain mechanisms of exteroceptive (e.g., visual, tactile, auditory) signals for BSC (Bekrater-Bodmann et al., 2012; Ehrsson et al., 2004; Ionta et al., 2011; Lenggenhager et al., 2011; Petkova et al., 2011; Tsakiris et al., 2007). The present patient data suggest that the processing of visual information with interoceptive signals, the present cardio-visual effects on BSC, is mediated by the insula. The insular cortex is part of a brain network involved in the processing of interoceptive and visceral information. Neuroimaging studies show that interoceptive signals are processed by the insula, anterior cingulate cortex, and somatosensory regions (Critchley et al., 2004; Pollatos et al., 2007; Wiebking et al., 2014; Zaki et al., 2012). Concerning the insula and BSC, we note that right insular activity has been associated with illusory ownership of a fake hand (Tsakiris et al., 2007) and that bilateral insula has been proposed to be a crucial structure for self-consciousness (Craig, 2002; Ionta et al., 2014). Neuropsychological studies also support the link between insula and BSC: patients with somatoparaphrenia, a delusional sense of disownership of contralesional body parts, may show right-brain damage involving the insula (Cereda et al., 2002; Karnath and Baier, 2010; Levine et al., 1991; Vallar and Ronchi, 2009 for a review). Other data indicate that caloric vestibular stimulation, a technique activating a network of brain regions 
including the insula (Bottini et al., 2001; Emri et al., 2003) may lead to a temporary remission of somatoparaphrenia (Bisiach et al., 1991; Rode et al., 1992). These studies and the present data converge on important contributions of the insula, in particular of the right insula, to BSC.

It is known that the insular cortex is related not only to interoceptive processing, but also to interoceptive awareness. In particular, right insular activity (mainly in the anterior insula) has been found to correlate with the level of interoceptive awareness of participants (Critchley et al., 2004; Pollatos et al., 2007). In line with these data, after resection of the right insular cortex, the present patient showed reduced, but not completely compromised, heartbeat awareness as detected with the Schandry heartbeat detection task. The persistence of interoceptive-exteroceptive (cardio-visual) integration, even if altered, could be explained by different brain mechanisms that are responsible for cardiac processing and awareness. It is well known that the right insula has a major role in interoceptive awareness, but the left insular cortex is also involved in interoceptive processing as shown by bilateral insular activations in several neuroimaging studies (Critchley et al., 2004; Wiebking et al., 2014; Zaki et al., 2012) and by the bilateral thalamo-insular projections of the interoceptive pathways (Craig, 2002). Additionally, it has been shown that insular and somatosensory regions constitute two different systems involved in cardiac awareness. Thus, the processing of interoceptive information is possible even after the bilateral lesion of insula (Khalsa et al., 2009); and in some conditions (e.g., in patients with external cardiac assist devices) somatosensory inputs can even dominate visceral ones for interoceptive awareness (Couto et al., 2014). These data support the finding of persisting (even if altered) processing of interoceptive information after right insular damage that is sufficient for the reported cardiovisual effects on BSC. Moreover, we note that the cardiac information conveyed in the present cardio-visual illusion paradigm is different from the one the patient was asked to pay attention to during the Schandry heartbeat detection task. In the first case, the cardiac information is externally shown as a visual stimulus with the same frequency of the heart. In the Schandry task, the request is to perceive the cardiac interoceptive signal, without the help of any external cue. Cardio-visual perception and cardiac perception do not refer to the same brain processes. One of these proposed mechanisms, or a combination of them, may have caused the enhanced illusory effect by cardio-visual signals in the insular patient, even after the resection of the tumour.

With respect to the performance of the healthy participants, previous behavioural studies suggest a link between interoceptive awareness and alteration of BSC, even if the direction of this relationship is still unclear. Concerning visuo-tactile own body illusions, previous work provided evidence in favour of a positive correlation (Suzuki et al., 2013), while others found evidence in favour of a negative correlation (Tajadura-Jiménez and Tsakiris, 2014; Tsakiris et al., 2011) between the level of interoceptive awareness and the level of experimentally induced changes in BSC. Complementarily, in an experimental manipulation of hand ownership by cardio-visual stimulation, Suzuki et al. (2013) reported a positive correlation between the level of awareness of cardio-visual synchrony and the level of illusory changes in BSC: in that study the measure of interoceptive awareness, which correlated with the cardio-visual effect, was the questionnaire's item investigating the explicit recognition of the synchrony of the visual feedback, while the correlation was not significant considering the heartbeat awareness task. The present study addresses this issue for the first time during the FBI. On the one hand, our results recorded in neurologically unimpaired participants are in line with those reported by Suzuki and collaborators: a tendency towards a significant positive correlation was found between self- identification and the questionnaire's item investigating the feeling that the flashing visual stimulus corresponded to participants' own heartbeat (Q9), while no significant correlation between the measures of heartbeat awareness and illusion was recorded. On the other hand, the data of the present patient demonstrate that BSC and the heartbeat detection task are differently linked to the right insula. Collectively, these data emphasize the relevance of interoceptive and exteroceptive signals for BSC and point to the role of the insula as an important structure for heartbeat awareness (Critchley et al., 2004; Pollatos et al., 2007) and BSC as mediated through interoceptive-exteroceptive conflicts. Moreover, even if BSC and heartbeat awareness are both linked to the activity of the insular cortex, interoceptive and exteroceptive information can be integrated and may elicit an alteration of BSC even with a reduced level of interoceptive awareness (as measured by the Schandry detection task).

Illusory reduplication of the body or body parts are complex clinical manifestations that have previously been described in the literature. The most common manifestations are autoscopic phenomena, which are illusory own body perceptions including outof-body experience, autoscopic hallucination and heautoscopy (Blanke and Mohr, 2005; Brugger, 2002; Devinsky et al., 1989). The most relevant of these phenomena for the present observation are neurological patients affected by heautoscopy: these patients report seeing a double of themselves in the extrapersonal space, associated with strong self-identification, and with frequent reports of bodily reduplication and of being at two places at the same time (bi-location: Brugger et al., 2006; Brugger, 2002; Heydrich and Blanke, 2013). We argue that the altered state of BSC characterized by changes in self-identification, self-location, as well as bi-location and bodily duplication in the present patient after cardio-visual stimulation shares many aspects with heautoscopy, suggesting that we may have induced a mild form of heautoscopy in the present patient through cardio-visual exposure with a virtual body. Feelings of body duplication and bi-location have also been reported in psychiatric (e.g., schizophrenia, depression, anxiety, dissociative disorders) and neurological (epilepsy, stroke, infection, migraine, neoplasia) diseases (Brugger et al., 1997; Bünning and Blanke, 2005; Dening and Berrios, 1994; Devinsky et al., 1989; Grüsser and Landis, 1991; Mohr and Blanke, 2005), and among them heautoscopy is frequently associated with emotional affinity towards the seen illusory body and depersonalization, as well as abnormal visceroceptive sensations (Brugger et al., 1997; Heydrich and Blanke, 2013). The present patient and heautoscopy patients also share insular damage, as brain damage in patients with heautoscopy was previously linked to the insula (Heydrich and Blanke, 2013). It has been argued that heautoscopy is caused by defective integration of visuo-somatosensory signals with emotional-interoceptive cues due to damage of the insular cortex (for a more detailed discussion see Heydrich and Blanke, 2013), further supported by evidence linking the insular cortex to emotional-social processing. Thus, previous studies showed that insular activity can be related to pain perception and observation of pain in other people (Singer et al., 2004), to see other people tasting pleasant and unpleasant drinks (Jabbi et al., 2007; see Singer et al., 2009 for a review), to self-other discrimination in face recognition (Kircher et al., 2000), in awareness of own actions (Corradi-Dell'Acqua et al., 2008; Farrer and Frith, 2002) and also in complex social interactions (Ebisch et al., 2011; Koban et al., 2013). More work is necessary to understand the differences that may exist between the involvement of right and left insula in interoception, interoceptive awareness, and their impact on BSC based on cardio-visual and visuo-tactile stimulations. Thus, the present patient suffered from right insular damage, whereas patients with heautoscopy were found to suffer from primarily left insular damage (Heydrich and Blanke, 2013). 
Another rare phenomenon (inner heautoscopy) that concerns the inner organs and that has been previously linked to heautoscopy (Blanke et al., 2004; Blanke and Mohr, 2005; Brugger et al., 1997) may be of relevance for the present patient. Inner heautoscopy is a rare illusory own body perception (Lhermitte, 1951; Menninger-Lerchenthal, 1935; Sollier, 1903; see also Dening and Berrios, 1994; Hécaen and Ajuriaguerra, 1952; Shilder, 1935) where patients claim to see an inner organ or part of an inner organ (heart, lungs, liver) in the exterapersonal space (for recent review see Blanke et al., 2008). Modern accounts of inner heautoscopy are rare (Carlson, 1977; Magrí and Mocchetti, 1967; Peto, 1969) and most cases have been described over hundred years ago (Bain, 1903; Comar, 1901; Sollier, 1903). With respect to the present patient, it is noteworthy that the heart seems to be the most prominent organ. Thus, Comar (1901; case \#1) described a 18year-old female patient who reported seeing her heart and Brugger et al. (1997) described a patient who saw the interior of his torso including heart and blood vessels. Sollier (1903) also described a patient claiming to see her heart, lungs, and other organs. Given the association of an alteration of heartbeat awareness and BSC (including the sensation of having two hearts) in the present patient with insular damage when exposed to cardio-visual stimulation, it may be suggested that inner heautoscopy may be a visualized (and thus externalized) percept based on abnormally integrated interoceptive and exteroceptive signals caused by insular damage. However, as reports about this condition are extremely rare and as there is currently no report in the literature about inner heautoscopy after acquired brain damage, caution is warranted and experimental data in such patients are needed.

Finally, we note that the growth of the tumour had a different impact on BSC and heartbeat awareness. Based on our results, the presence of the tumour, before its resection, mainly affected BSC, with cardio-visual stimulation triggering illusory self-identification (in a not synchrony-specific fashion), body-duplication and bi-location. Thus, BSC was already altered in the pre-surgical phase. Related alteration of BSC have been reported in previous studies, such as heautoscopy and body reduplication, in which these manifestations were observed during unilateral brain tumour infiltration (Brugger et al., 2006) or in regions with unilateral epileptic seizures and thus without focal damage or brain tissue removal (Heydrich and Blanke, 2013). Based on these data, we argue that insular dysfunction without complete insular damage is sufficient to alter multisensory integration between cardiac and visual signals that are of relevance for BSC, emotional processing and self-other discrimination. These processes most likely require a functional network of brain regions beyond the insula: by consequence, in the presence of a pathology affecting the insula (a key brain area for the function of interest), ad hoc stimulation (including cardio-visual behavioural stimulations, as in our case, or abnormal excessive neuronal activity in the brain, as during epileptic seizure) may elicit the altered BSC phenomena (related to heautoscopy) that we describe. We also note that we have detected small differences in BSC alterations evoked before and after the surgery: changes in self-location and feeling of bi-location were equally induced in the pre- and post-surgery evaluations; however, after tumour removal the illusory effects on BSC became synchrony-dependent for self-identification and did not involve body reduplication (as shown by the statistical comparison between patient and controls). We note, however, that these findings still remain selective as control items were similarly rated before and after the surgery. Self-location (i.e., the experience of where I am in space) and self-identification (i.e., the experience of owning a body) refer to two different, even if closely linked, aspects of BSC; moreover, it has been experimentally demonstrated that the manipulation of bodily signals may alter selectively one or more of these characteristics (see Blanke, 2012 for a review). By consequence, two types of brain impairment (i.e., dysfunctional brain caused by tumour infiltration versus complete structural brain damage by surgical resection) may affect in a slightly different way these two components of BSC. In particular, with respect to self-identification, the presence of a strong, synchronyindependent effect before surgery could be caused by the recruitment of dysfunctional tissue within the right insular region for cardio-visual processing, with an undiscriminating illusory outcome for synchronous and asynchronous signals. An alternative may be that these dissimilarities are caused by differences in anti-epileptic medication, with less efficient treatment in the early pre-surgery evaluation (i.e., 5 days after anti-epileptic treatment was started).

Differing from BSC, heartbeat awareness was not affected by tumour growth, compared to age-matched controls. We argue that this is related to the presence of functional vicarious brain regions beyond the right insula, compensating for interoceptive awareness. Thus, previous studies have demonstrated that in patients with low grade gliomas some structures may still remain functional within brain regions infiltrated by a tumour; moreover, it has been shown that peri-lesional areas can be recruited and can compensate for impaired functions during phases of progressive tumour growth (Duffau et al., 2003; Schiffbauer et al., 2001), differing from the effects of acute brain damage due to stroke or neurosurgical resection. Accordingly, we argue that the present post-operative reduction of heartbeat awareness was detected because the surgical removal of the brain tumour included also those structures that were functional within the tumour and the surrounding tissue. We propose that this may explain why heartbeat awareness in our patient was still preserved with respect to controls before the surgery and affected after the tumour removal. However, we note that, although after tumour resection the heartbeat awareness level of the patient was significantly decreased with respect to the pre-surgery level, it was still not significantly different from the average heartbeat awareness of the neurologically unimpaired participants (i.e., it was comparable with the low-level sub-group): again, this could be accounted for by the presence of functional regions beyond the area of resection (Duffau, 2014) not damaged or removed by the surgery and, furthermore, argue for the existence of other brain regions playing an important role in interoceptive awareness (in addition to the right insula, such as bilateral somatosensory cortical networks) (Couto et al., 2014; Khalsa et al., 2009).

In conclusion, the present case report corroborates the key role of the insula in interoceptive-cardiac processing, heartbeat awareness, and presents the first evidence of its involvement in mediating cardio-visual effects that have been shown to impact BSC. Moreover, in the case of insular damage this BSC alteration may be associated with a loss of the experienced unity of the self, perceived as bi-location and body reduplication.

\section{Acknowledgements}

Olaf Blanke is supported by the Bertarelli Foundation, the Swiss National Science Foundation (\#51AU40_125759), the Roger de Spoelberch foundation and the National Center of Competence in Research - Synapsy (The Synaptic Bases of Mental Diseases). Roberta Ronchi is supported by the EPFL international fellowship program co-founded by the European Union's Seventh Framework Programme (Grant agreement no. 291771).

\section{References}

Aspell, J.E., Heydrich, L., Marillier, G., Lavanchy, T., Herbelin, B., Blanke, O., 2013. Turning body and self inside out: visualized heartbeats alter bodily self- 
consciousness and tactile perception. Psychol. Sci. 24 (12), 2445-2453.

Aspell, J.E., Lenggenhager, B., Blanke, O., 2009. Keeping in touch with one's self: multisensory mechanisms of self-consciousness. PloS One 4 (8), e6488.

Bain, A., 1903. De l'autorepresentation chez les hysteriques. Vigot, Paris.

Bekrater-Bodmann, R., Foell, J., Diers, M., Flor, H., 2012. The perceptual and neuronal stability of the rubber hand illusion across contexts and over time. Brain Res. 1452, 130-139.

Bisiach, E., Rusconi, M.L., Vallar, G., 1991. Remission of somatoparaphrenic delusion through vestibular stimulation. Neuropsychologia 29 (10), 1029-1031.

Blanke, O., 2012. Multisensory brain mechanisms of bodily self-consciousness. Nat. Rev. Neurosci. 13 (8), 556-571.

Blanke, O., Arzy, S., Landis, T., 2008. Illusory reduplications of the human body and self. Handb. Clin. Neurol. 88, 429-458.

Blanke, O., Landis, T., Spinelli, L., Seeck, M., 2004. Out-of-body experience and autoscopy of neurological origin. Brain 127 (Part 2), 243-258.

Blanke, O., Metzinger, T., 2009. Full-body illusions and minimal phenomenal selfhood. Trends Cognit. Sci. 13 (1), 7-13.

Blanke, O., Mohr, C., 2005. Out-of-body experience, heautoscopy, and autoscopic hallucination of neurological origin Implications for neurocognitive mechanisms of corporeal awareness and self-consciousness. Brain Res. Rev. 50 (1), 184-199.

Bloesch, E.K., Davoli, C.C., Abrams, R.A., 2013. Age-related changes in attentional reference frames for peripersonal space. Psychol. Sci. 24 (4), 557-561.

Bolognini, N., Ronchi, R., Casati, C., Fortis, P., Vallar, G., 2014. My hand is back! Multisensory remission of somatoparaphrenic delusion. Neurol.: Clin. Pract. 4 (3), 216-225.

Bottini, G., Karnath, H.O., Vallar, G., Sterzi, R., Frith, C.D., Frackowiak, R.S., Paulesu, E., 2001. Cerebral representations for egocentric space: functional-anatomical evidence from caloric vestibular stimulation and neck vibration. Brain 124 (Part 6), 1182-1196.

Broughton, R., Wasel, N., 1990. A text-stimuli presentation manager for the IBM PC with ipsatization correction for response sets and reaction times. Behav. Res. Methods Instrum. Comput. 22 (4), 421-423.

Brugger, P., 2002. Reflective mirrors: perspective-taking in autoscopic phenomena. Cognit. Neuropsychiatry 7 (3), 179-194.

Brugger, P., Blanke, O., Regard, M., Bradford, D.T., Landis, T., 2006. Polyopic heautoscopy: Case report and review of the literature. Cortex 42 (5), 666-674.

Brugger, P., Regard, M., Landis, T., 1997. Illusory reduplication of one's own-body: phenomenology and classification of autoscopic phenomena. Cognit. Neuropsychiatry $2,19-38$.

Bünning, S., Blanke, O., 2005. The out-of body experience: precipitating factors and neural correlates. Prog. Brain Res. 150, 331-350.

Caçola, P., Martinez, A., Ray, C., 2013. The ability to modulate peripersonal and extrapersonal reach space via tool use among the elderly. Arch. Gerontol. Geriatr. 56 (2), 383-388.

Carlson, D.A., 1977. Dream mirrors. Psychoanal. Q. 46 (1), 38-70.

Cattell, R., 1944. Psychological measurement: normative, ipsative, interactive. Psychol. Rev. 51 (5), 292-303.

Cereda, C., Ghika, J., Maeder, P., Bogousslavsky, J., 2002. Strokes restricted to the insular cortex. Neurology 59 (12), 1950-1955.

Comar, G., 1901. L'auto-représentation de l'organisme chez quelques hystériques. Rev. Neurol. 9, 490-495.

Corradi-Dell'Acqua, C., Ueno, K., Ogawa, A., Cheng, K., Rumiati, R.I., Iriki, A., 2008. Effects of shifting perspective of the self: an fMRI study. Neurolmage 40 (4), 1902-1911.

Couto, B., Salles, A., Sedeño, L., Peradejordi, M., Barttfeld, P., Canales-Johnson, A., Ibanez, A., 2014. The man who feels two hearts: the different pathways of interoception. Soc. Cognit. Affect. Neurosci. 9 (9), 1253-1260.

Craig, A.D., 2002. How do you feel? Interoception: the sense of the physiological condition of the body. Nat. Rev. Neurosci. 3 (8), 655-666.

Crawford, J.R., Garthwaite, P.H., 2002. Investigation of the single case in neuropsychology: confidence limits on the abnormality of test scores and test score differences. Neuropsychologia 40 (8), 1196-1208.

Critchley, H.D., Wiens, S., Rotshtein, P., Ohman, A., Dolan, R.J., 2004. Neural systems supporting interoceptive awareness. Nat. Neurosci. 7 (2), 189-195.

Damasio, A., 2000. The Feeling of What Happens: Body and Emotion in the Making of Consciousness. Harcourt Brace, New York.

Dening, T.R., Berrios, G.E., 1994. Autoscopic phenomena. Br. J. Psychiatry: J. Ment. Sci. 165 (6), 808-817.

De Vignemont, F., 2011. Embodiment, ownership and disownership. Conscious. Cognit. 20 (1), 82-93.

Devinsky, O., Feldmann, E., Burrowes, K., Bromfield, E., 1989. Autoscopic phenomena with seizures. Arch. Neurol. 46 (10), 1080-1088.

Duffau, H., 2014. Diffuse low-grade gliomas and neuroplasticity. Diagn Interv. Imaging 95 (10), 945-955.

Duffau, H., Capelle, L., Denvil, D., Sichez, N., Gatignol, P., Lopes, M., Van Effenterre, R. 2003. Functional recovery after surgical resection of low grade gliomas in eloquent brain: hypothesis of brain compensation. J. Neurol. Neurosurg. Psychiatry 74 (7), 901-907.

Ebisch, S.J.H., Ferri, F., Salone, A., Perrucci, M.G., D’Amico, L., Ferro, F.M., Gallese, V. 2011. Differential involvement of somatosensory and interoceptive cortices during the observation of affective touch. J. Cognit. Neurosci. 23 (7), 1808-1822.

Ehrsson, H.H., 2007. The experimental induction of out-of-body experiences. Science 317 (5841), 1048.

Ehrsson, H.H., Spence, C., Passingham, R.E., 2004. That's my hand! Activity in premotor cortex reflects feeling of ownership of a limb. Science 305 (5685),
$875-877$.

Emri, M., Kisely, M., Lengyel, Z., Balkay, L., Márián, T., Mikó, L., Tóth, A., 2003. Cortical projection of peripheral vestibular signaling. J. Neurophysiol. 89 (5), 2639-2646.

Farrer, C., Frith, C.D., 2002. Experiencing oneself vs another person as being the cause of an action: the neural correlates of the experience of agency. NeuroImage 15 (3), 596-603.

Ghafouri, M., Lestienne, F.G., 2000. Altered representation of peripersonal space in the elderly human subject: a sensorimotor approach. Neurosci. Lett. 289 (3), 193-196.

Grüsser, O.-J., Landis, T., 1991. The splitting of "I" and "me": heautoscopy and related phenomena In: Grüsser, O.J., Landis, T. (Eds.), Visual Agnosias and other Disturbances of Visual Perception and Cognition, pp. 297-303.

Hécaen, H., Ajuriaguerra, J. de, 1952. L'Heautoscopie, Meconnassiances et hallucinations corporelles. Masson, Paris.

Heydrich, L., Blanke, O., 2013. Distinct illusory own-body perceptions caused by damage to posterior insula and extrastriate cortex. Brain 136 (Part 3), 790-803.

Heydrich, L., Dodds, T.J., Aspell, J.E., Herbelin, B., Bülthoff, H.H., Mohler, B.J., Blanke, O., 2013. Visual capture and the experience of having two bodies - evidence from two different virtual reality techniques. Front. Psychol. 4, 946.

Ionta, S., Heydrich, L., Lenggenhager, B., Mouthon, M., Fornari, E., Chapuis, D. Blanke, O., 2011. Multisensory mechanisms in temporo-parietal cortex support self-location and first-person perspective. Neuron 70 (2), 363-374.

Ionta, S., Martuzzi, R., Salomon, R., Blanke, O., 2014. The brain network reflecting bodily self-consciousness: a functional connectivity study. Soc. Cognit. Affect. Neurosci. 9 (12), 1904-1913.

Jabbi, M., Swart, M., Keysers, C., 2007. Empathy for positive and negative emotions in the gustatory cortex. NeuroImage 34 (4), 1744-1753.

Karnath, H.-O., Baier, B., 2010. Right insula for our sense of limb ownership and selfawareness of actions. Brain Struct. Funct. 214 (5-6), 411-417.

Khalsa, S.S., Rudrauf, D., Feinstein, J.S., Tranel, D., 2009. The pathways of interoceptive awareness. Nat. Neurosci. 12 (12), 1494-1496.

Kircher, T.T., Senior, C., Phillips, M.L., Benson, P.J., Bullmore, E.T., Brammer, M., David, A.S., 2000. Towards a functional neuroanatomy of self processing: effects of faces and words. Brain Res. Cognit. Brain Res. 10 (1-2), 133-144.

Koban, L., Corradi-Dell'Acqua, C., Vuilleumier, P., 2013. Integration of error agency and representation of others' pain in the anterior insula. J. Cognit. Neurosci. 25 (2), 258-272.

Lenggenhager, B., Halje, P., Blanke, O., 2011. Alpha band oscillations correlate with illusory self-location induced by virtual reality. Eur. J. Neurosci. 33 (10), 1935-1943.

Lenggenhager, B., Tadi, T., Metzinger, T., Blanke, O., 2007. Video ergo sum: manipulating bodily self-consciousness. Science 317 (5841), 1096-1099.

Lepelley, M.-C., Thullier, F., Bolmont, B., Lestienne, F.G., 2010. Age-related differences in sensorimotor representation of space in drawing by hand. Clin. Neurophysiol.: Off. J. Int. Fed. Clin. Neurophysiol. 121 (11), 1890-1897.

Levine, D.N., Calvanio, R., Rinn, W.E., 1991. The pathogenesis of anosognosia for hemiplegia. Neurology 41 (11), 1770-1781.

Lhermitte, J., 1951. Les phenomènes héautoscopiques, les hallucinations spéculaires In: Doin, G., Cie (Eds.), Les Hallucinations. Clinique et Physiopathologie, Paris, pp. $124-168$.

Magrí, R., Mocchetti, E., 1967. [Partial asomatoscopy (negative autoscopy) in epileptics. Nosographic classification and clinical contribution]. Arch. Psicol. Neurol. Psichiatr. 28 (6), 572-585.

Mahoney, J.R., Li, P.C.C., Oh-Park, M., Verghese, J., Holtzer, R., 2011. Multisensory integration across the senses in young and old adults. Brain Res. 1426, 43-53.

Menninger-Lerchenthal, E., 1935. Das Truggebilde der eigenen Gestalt. Karger, Berlin.

Mohr, C., Blanke, O., 2005. The demystification of autoscopic phenomena: experimental propositions. Curr. Psychiatry Rep. 7 (3), 189-195.

Palluel, E., Aspell, J.E., Blanke, O., 2011. Leg muscle vibration modulates bodily selfconsciousness: integration of proprioceptive, visual, and tactile signals. J. Neurophysiol. 105 (5), 2239-2247.

Petkova, V.I., Björnsdotter, M., Gentile, G., Jonsson, T., Li, T.-Q., Ehrsson, H.H., 2011. From part- to whole-body ownership in the multisensory brain. Curr. Biol. 21 (13), 1118-1122.

Petkova, V.I., Ehrsson, H.H., 2008. If I were you: perceptual illusion of body swapping. PloS One 3 (12), e3832.

Peto, A., 1969. Terrifying eyes. A visual superego forerunner. Psychoanal. Study Child 24, 197-212.

Pollatos, O., Schandry, R., Auer, D.P., Kaufmann, C., 2007. Brain structures mediating cardiovascular arousal and interoceptive awareness. Brain Res. 1141, 178-187.

Rode, G., Charles, N., Perenin, M.T., Vighetto, A., Trillet, M., Aimard, G., 1992. Partial remission of hemiplegia and somatoparaphrenia through vestibular stimulation in a case of unilateral neglect. Cortex 28 (2), 203-208.

Romano, D., Pfeiffer, C., Maravita, A., Blanke, O., 2014. Illusory self-identification with an avatar reduces arousal responses to painful stimuli. Behav. Brain Res. 261, 275-281.

Schandry, R., 1981. Heart beat perception and emotional experience. Psychophysiology 18 (4), 483-488.

Schiffbauer, H., Ferrari, P., Rowley, H.A., Berger, M.S., Roberts, T.P., 2001. Functional activity within brain tumors: a magnetic source imaging study. Neurosurgery 49 (6), 1313-1320

Shilder, P., 1935. The Image and Appearance of the Human Body. Georg Routledge and Sons, Regan Paul Trench, Trubner \& Co, London.

Siegel, S., Castellan, N.J., 1988. Nonparametric Statistics for the Behavioral Sciences. 
McGraw-Hill, New York.

Singer, T., Critchley, H.D., Preuschoff, K., 2009. A common role of insula in feelings, empathy and uncertainty. Trends Cognit. Sci. 13 (8), 334-340.

Singer, T., Seymour, B., O’Doherty, J., Kaube, H., Dolan, R.J., Frith, C.D., 2004. Empathy for pain involves the affective but not sensory components of pain. Science 303 (5661), 1157-1162.

Sollier, P., 1903. L'autoscopie interne. Rev. Philos. 55, 1-41.

Suzuki, K., Garfinkel, S.N., Critchley, H.D., Seth, A.K., 2013. Multisensory integration across exteroceptive and interoceptive domains modulates self-experience in the rubber-hand illusion. Neuropsychologia 51 (13), 2909-2917.

Tajadura-Jiménez, A., Tsakiris, M., 2014. Balancing the "inner" and the "outer" self: interoceptive sensitivity modulates self-other boundaries. J. Exp. Psychol. Gen. 143 (2), 736-744.

Tsakiris, M., Hesse, M.D., Boy, C., Haggard, P., Fink, G.R., 2007. Neural signatures of body ownership: a sensory network for bodily self-consciousness. Cereb. Cortex 17 (10), 2235-2244.

Tsakiris, M., Tajadura-Jiménez, A., Costantini, M., 2011. Just a heartbeat away from one's body: interoceptive sensitivity predicts malleability of body-representations. Proc. Biol. Sci./R. Soc. 278 (1717), 2470-2476.

Vallar, G., Ronchi, R., 2009. Somatoparaphrenia: a body delusion. A review of the neuropsychological literature. Exp. Brain Res. 192 (3), 533-551.

Wiebking, C., Duncan, N.W., Tiret, B., Hayes, D.J., Marjaǹska, M., Doyon, J., Northoff, G., 2014. GABA in the insula-a predictor of the neural response to interoceptive awareness. NeuroImage 86, 10-18.

Zaki, J., Davis, J.I., Ochsner, K.N., 2012. Overlapping activity in anterior insula during interoception and emotional experience. NeuroImage 62 (1), 493-499. 